\title{
Usability of a telehealth solution based on TV interaction for the elderly: the VITASENIOR-MT case study
}

\author{
Gabriel Pires ${ }^{1}\left(\mathbb{D} \cdot\right.$ Ana Lopes $^{1} \cdot$ Pedro Correia $^{1} \cdot$ Luis Almeida $^{1} \cdot$ Luis Oliveira $^{1} \cdot$ Renato Panda $^{1} \cdot$ Dario Jorge $^{1}$. \\ Diogo Mendes ${ }^{1} \cdot$ Pedro Dias ${ }^{1} \cdot$ Nelson Gomes ${ }^{1} \cdot$ Telmo Pereira $^{2}$
}

Accepted: 29 November 2021

○ The Author(s), under exclusive licence to Springer-Verlag GmbH Germany, part of Springer Nature 2021

\begin{abstract}
Remote monitoring of biometric data in the elderly population is an important asset for improving the quality of life and level of independence of elderly people living alone. However, the design and implementation of health technological solutions often disregard the elderly physiological and psychological abilities, leading to low adoption of these technologies. We evaluate the usability of a remote patient monitoring solution, VITASENIOR-MT, which is based on the interaction with a television set. Twenty senior participants (over 64 years) and a control group of 20 participants underwent systematic tests with the health platform and assessed its usability through several questionnaires. Elderly participants scored high on the usability of the platform, very close to the evaluation of the control group. Sensory, motor and cognitive limitations were the issues that most contributed to the difference in usability assessment between the elderly group and the control group. The solution showed high usability and acceptance regardless of age, digital literacy, education and impairments (sensory, motor and cognitive), which shows its effective viability for use and implementation as a consumer product in the senior market.
\end{abstract}

Keywords Telehealth $\cdot$ Remote patient monitoring $\cdot$ TV interaction $\cdot$ Usability $\cdot$ User-centred design $\cdot$ Elderly $\cdot$ Biometric and environmental data $\cdot$ Blood pressure $\cdot$ Glycaemia $\cdot$ Weight $\cdot$ Heart rate $\cdot$ Oximetry

\section{Introduction}

In the European Union, the life expectancy in 2016 was 81 years, 3.3 years more than in 2002. It is estimated that the old age dependency ratio will result in a decrease from 3.1 workers/1 pensioner to 2 workers/1 pensioner in 2070 [1]. This demographic scenario is creating a significant demand in healthcare and social systems, which need to be efficient, sustainable and as close as possible to the population. In this scenario, the elderly appear as one of the most vulnerable groups because of the increased comorbidity, and also due to

This work has been financially supported by the Portuguese foundation for science and technology (FCT) and European funds through Project VITASENIOR-MT with grant CENTRO-01-0145FEDER-023659.

Gabriel Pires

gppires@ipt.pt

1 Polytechnic Institute of Tomar, Lab. VITA.IPT, 2300-313 Tomar, Portugal

2 School of health technology, Polytechnic Institute of Coimbra, 3045-093 Coimbra, Portugal difficulties in accessing informal and institutional care, usually associated with a fragile socioeconomic condition [2]. In most countries, cardiovascular and oncological diseases constitute the most significant health burden in older adults, and multimorbidity is also common, leading to complex polimedication regimens.

Several technological approaches have been proposed in the last decades, either to promote healthy and active ageing, or to provide instruments to monitor and assist the independent and autonomous living of older adults. Considering the latter, several remote patient monitoring (RPM) solutions to avoid the institutionalization and to promote independent living have been developed and tested. The Covid-19 pandemic has also highlighted the importance of RPM and how it can be used to safely provide care. These technologies consist mainly of safety and security monitoring of health, vital signs and environmental parameters [3]. However, most studies evaluating these solutions highlight the need for technological solutions amenable to tailored configurations that meet individual needs (see survey in [4]). In particular, the ageing population has unique needs resulting from the progressive deterioration of physiological and psychological 
abilities, which leads to low adoption of health technologies when the characteristics and idiosyncrasies of these users are disregarded in the design and development of products [5, 6]. In terms of human-computer interaction, many technological, social and cultural factors have also been shown to limit the deployment of healthcare technologies, as surveyed in [7].

Some telehealth services are already well established, such as teleconsultation [8], telemedicine [9] and emergency alert systems (telecare) [10] based on technologies that have been widely implemented for several decades (e.g. telephone, videoconferencing). However, commercial services for remote monitoring of biometric parameters for the elderly population are still incipient, in part due to the low usability of current systems, given the particular needs and skills of the elderly. Remote monitoring can play an important role in supporting medical diagnosis, surveillance of patients in home hospitalization during subacute periods [11] or for continuous monitoring of people with chronic diseases, complementing patients' self-management at home [12], for example, through monitoring of weight, heart rate, oxygen saturation, blood pressure and glycaemia [13]. Yet, services to transfer these data to remote systems mostly depend on the use of smartphone or tablet applications (mHealth scenarios) [14], which represents a barrier to their use by the majority of older adults [15]. The digital literacy of the elderly is very dependent on their social, cultural and economic condition, but in general is low [5, 16]. Seniors are quite resistant to new technologies or have difficulty in using them due to vision, hearing, sensory, dexterity and cognition impairments [15, 17-19]. To overcome these difficulties, and to guarantee the success of a given application, the interaction models and technologies to be considered must be adapted to this reality, aiming at simpler, functional and useful approaches [20]. In this perspective, the use of TV sets has been proposed as an easy-to-use technological solution that offers some advantageous features and may increase technology acceptance. Namely, it has a familiar interface, and therefore, the elderly are used to control their basic functions, the screen is large enough to show large text and icons, it is very easy to adjust the audio level, and finally, almost everyone has one, thus reducing the additional charge [6, 21-26].

\subsection{TV-based interaction systems for senior health care and info-inclusion}

Several proof-of-concept TV-based systems have been proposed in remote biometric monitoring contexts. With the development of digital television, some first experiments soon emerged to integrate TV-based interactive services. Gork et al. [27] proposed one of the first related works, an interactive digital television framework considering usability concerns for elderly users. The system provided a customized user interface that adapted to the needs of each user group. Videoconference and informational services were tested with 83 users with promising results. In [28], a digital video broadcasting terrestrial multimedia home platform (DVBT-MHP) connected to the TV was used for health monitoring (level of glucose in the blood, oximetry, blood pressure, heart rate and body weight). This work has shown the potential of TV-based remote monitoring including user authentication and data security issues. However, it was highly dependent on digital terrestrial television infrastructure, which limits its deployment. For instance, MHP and DVBT return channel services are not available in many countries, as is the case in Portugal. With the rise of smart TVs, web-based set-top boxes and Android TVs, new services have been proposed, allowing integration with web and cloud services, opening new perspectives for user interfaces adapted to the elderly population. In [25], the authors proposed an open model architecture (open home theatre personal computer (HTPC) set-top box (STB)) to support existing health and social services, which can be deployed in Smart TVs. Preliminary non-systematic tests with a diverse set of applications (including blood pressure monitoring) were carried out with 62 seniors. The iTVcare platform proposed in [29] has been tested by a group of 50 older adults to assess seniors' acceptance of a TV-based solution used for medical reminders (appointments and medication intake). The HEREiAM platform proposed in [24] used an Androidbased system supported by a private cloud system and was designed to be accessible both via TV and portable devices, which is an important asset of the system. The platform connects medical devices via Bluetooth and includes some other useful services such as the weather forecast or services designed to support the elderly living alone. The platform has been tested at different times by a group of 19 older adults, although with a limited number of medical devices (blood pressure and body weight).

TV-based systems were also proposed in different contexts. For example, in [21] a combined TV-computer solution was proposed to explore the idea of a "hidden" computer behind the TV console providing the main functionalities of a computer. The system was tested by a group of 14 seniors. In [26], an interactive platform, running on an Android STB, was developed and tested by 8 participants over 60 years old to promote the info-inclusion in the elderly population, providing informative contents about useful services, previously obtained via focus groups. Its current application is somehow limited since it is based only on open streaming TV channels. Commercial solutions are already available to offer social interaction, like photo sharing and videoconferencing, social activities, teleconsultation and health reminders [30, 31]. 
The promising results already obtained with TV-based systems with the senior population emphasize the viability of this type of solutions, which are still relatively rare. In particular, for TV-based remote biometric monitoring, there are no commercially available solutions.

\subsection{VITASENIOR-MT healthcare solution}

In this context, we previously proposed VITASENIORMT telehealth solution, a platform that does remote monitoring of biometric and environmental parameters [32]. The solution was specifically designed for a senior population with low digital literacy and considering the physical and cognitive limitations that they may present. Thus, both system's easiness of use and learning curve were key aspects considered during the development. At the same time, the solution was designed to be highly scalable in a perspective of gradually adding new heterogeneous medical and environmental devices, but also from a perspective of the number of home users (customers), always ensuring safety, security and privacy of data [33].

In [32] and [33], we focused on the implementation details and the validation of system stability from a hardware and software perspective. In the current paper, the main goal is to evaluate the usability of the TV-based VITASENIOR-MT solution as a telehealth platform. We report the experimental evaluation of the solution by end-users, considering its usefulness, satisfaction, ease of use and ease of learning. The solution was designed and validated following a user-centred design (UCD) perspective along 3 phases. The first phase was used to collect users' feedback about the system and to adjust it accordingly (this period was longitudinal to the project development). In the second phase, the system was systematically assessed by a group of 20 seniors from elderly care centres and a control group of 20 people outside those centres, who answered to questionnaires assessing usefulness, satisfaction, ease of use and ease of learning. Then, three participants, selected from the senior group, used the home-care platform for two months. The home-care platforms were installed in 2 different senior centres (nursing homes), where experimental tests were conducted with the elderly and health professionals (doctors and nurses of the institutions). The main contribution of the paper is to set forth the viability of the interactive telehealth solution via TV for the elderly population. The solution showed a high usability regardless of age, digital literacy, education and impairments (sensory, motor and cognitive), which shows its effective potential for use and implementation in the senior market.

\section{VITASENIOR-MT architecture}

VITASENIOR-MT was designed as a highly scalable telehealth IoT platform that remotely monitors biometric and environmental data. The system comprises four subsystems: a home-care platform based on a TV set for interaction with direct end-users, a wireless sensor network (WSN) for environmental data collection, a cloud infrastructure following a microservice and fog-computing architecture and a web portal for interaction with formal and informal caregivers. The overall architecture is shown in Fig. 1, and the functional parts concerning user-interaction will be described in the following sub-sections highlighting the functionality, reliability and usability of the provided services (technical implementation details regarding the Cloud infrastructure, security and privacy mechanisms are not included in this paper, as they were already reported in [32] [33]).

\subsection{Home-care telehealth platform and WSN}

The core of the telehealth home-care platform is based on a Raspberry Pi 3 (RPi3) micro-computer integrated into a hub-box called VITABOX, which is responsible for interacting with a TV, Bluetooth medical devices, IEEE 802.15.4 WSN, and the cloud infrastructure. The RPi3 has all the required communication modules, namely Bluetooth, WLAN IEEE 802.11, HDMI-CEC (consumer electronic control) and USB to connect to the IEEE 802.15.4 WSN border router (inside VITABOX). Both VITABOX and the set-top box receiver are connected to the TV set through 2 HDMI ports, making the telehealth approach independent of the television service provider. Toggling between these two modes occurs either manually, at the user's own initiative, using the TV's remote control, or automatically, when alarm situations are triggered by abnormal values of environmental sensors. The interaction of the direct end-user with the telehealth system is done through the TV's remote control. This feature was a key design and implementation requirement of the VITASENIOR-MT system, which aims to be familiar, functional, easy to use and requiring the minimum number of steps as possible to perform the telehealth tasks. The end-user accesses all features of the home-care platform through 7 keys of the remote, namely, "OK", "L/R/U/D Arrows", "Back" and "1", as depicted in Fig. 2. The "1" key is used to toggle between TV and telehealth modes, by user's own initiative. If the user receives a notification at the lower right corner of the TV while watching TV (see Fig. 1), the TV also toggles to telehealth mode by pressing the key "OK" and shows the notification screen. In telehealth 


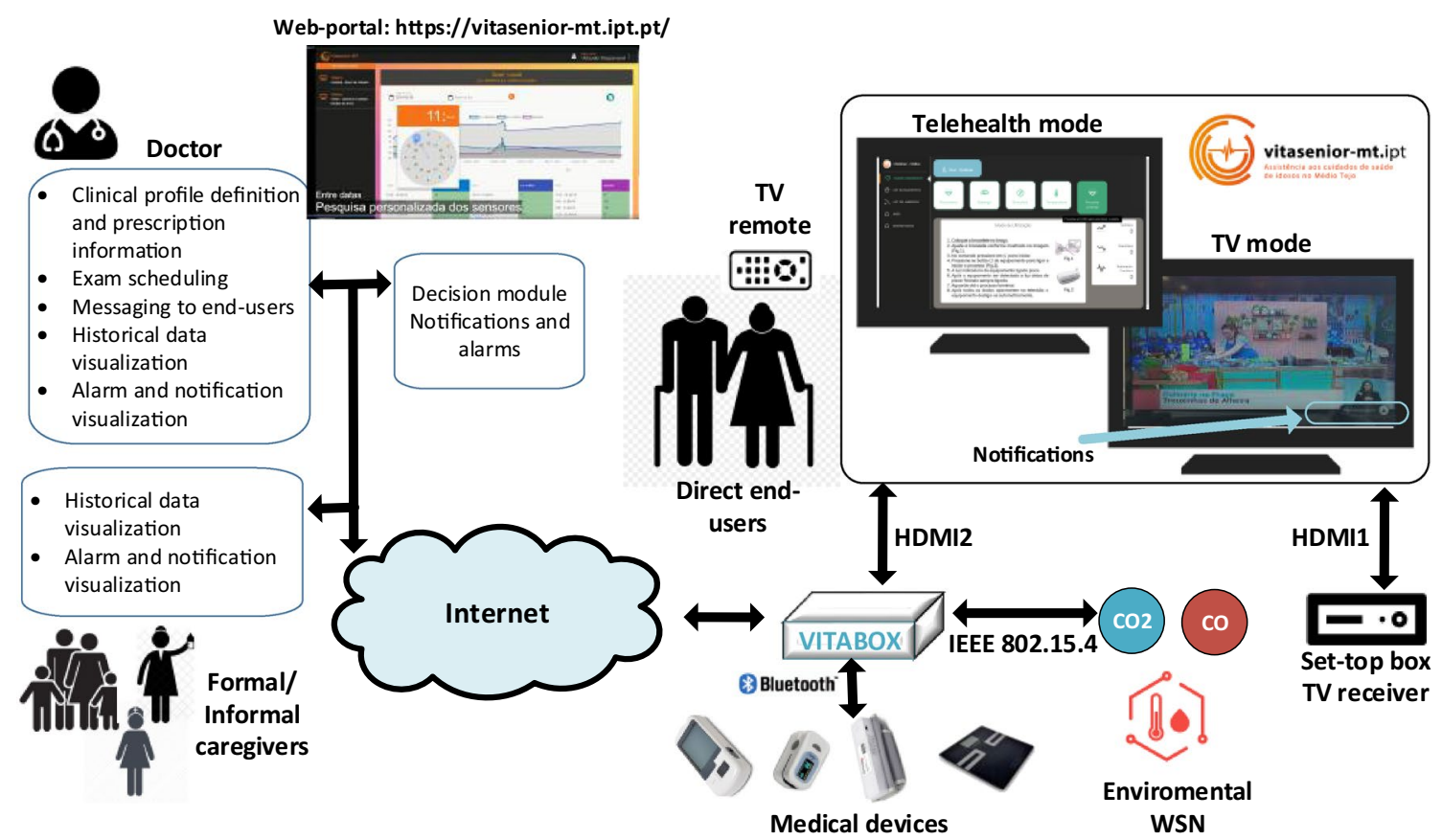

Fig. 1 VITASENIOR-MT architecture overview

Fig. 2 a End-user interacting with the VITASENIOR-MT home-care platform to measure the blood pressure; $\mathbf{b}$ keys of the $\mathrm{TV}$ remote used for interaction with the TV application

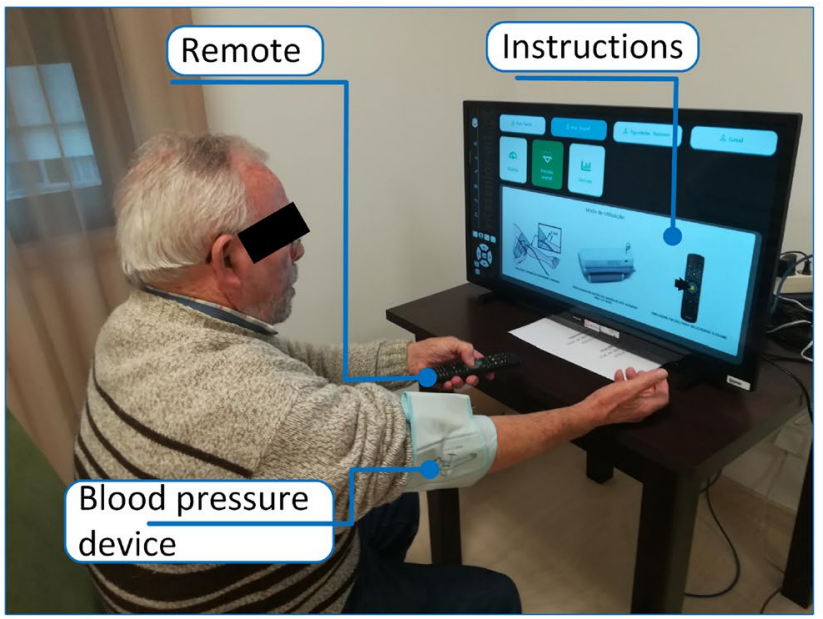

a)

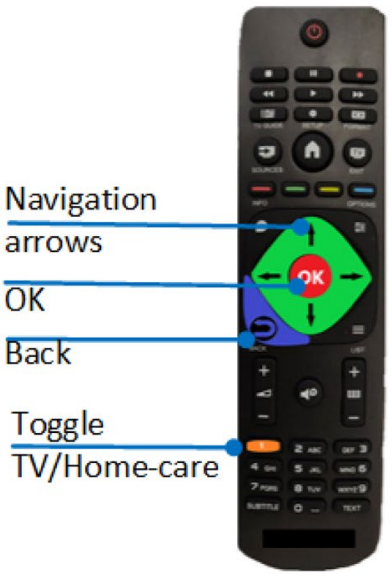

b) mode, the user has an initial welcome screen with information regarding the day, date and time, weather information and a list of the open pharmacies nearby. From the sidebar menu, the user can access the main options such as "Exams", "Exam history" and "Environmental history" using the remote's arrow keys (see demo videos ${ }^{1}$ ). After choosing the desired option, the user selects at the top of the screen the tab associated with his/her profile, since

\footnotetext{
${ }^{1} \mathrm{http} / / /$ orion.ipt.pt/ gpires/VITASENIOR-MT/videos/videos.htm
}

more than one user can be registered in the same platform (e.g. a couple). By selecting the "Exams" sidebar option, a set of medical devices associated with the user's profile is exhibited. Currently, the following medical devices are available: blood pressure meter, glucometer, oximeter and weight scale (heart rate is included in the blood-pressure meter and the oximeter). Each medical device is selected by pressing the remote's "OK" key. The "instructions of use" are image- and text-based, accompanied by audiodescription. Each medical device has its own way of operation, like any other regular device. For example, a blood 
pressure measurement requires pressing the start button, whereas for the weight scale the user only needs to step on the scale. The communication between VITABOX and the device occurs in a transparent way, without any intervention from the user. At the end of the measurement, the data are transferred to the VITABOX and communicated to the user in a visual and auditory way. Visual layout, audio texts and interaction procedures were all adjusted based on the feedback of end-users and professional and informal caregivers, during the first phase of the usability tests in a UCD perspective, as described in sect. 3. Environmental data $(\mathrm{CO}, \mathrm{CO} 2$, temperature and humidity) are collected from the WSN transparently and made available for visualization on the TV. Implementation details can be found in [32].

\subsection{VITASENIOR-MT web portal}

VITASENIOR-MT web portal was implemented as a progressive web application (PWA), allowing it to be installed similarly to a native application in any device that runs a web browser, making access to the platform easier. It was designed considering usability requirements that could serve users with different experience levels, who in most cases are not familiar with new technologies. All VITASENIORMT users with an account can interact with the web portal, namely doctors, formal caregivers (nurses, geriatric nursing assistants) and informal caregivers (family members) and direct end-users. On the other hand, the system was built ensuring the installation of the home platform's kit was as plug and play as possible (that includes the VITABOX, medical devices, and environmental sensors). This is done by pre-registering all equipment, still inactive, which will be available for installation.

The user responsible for the VITABOX (e.g. a relative of the end-user, hereinafter referred to as sponsor) is able to acquire and associate additional equipment to his/her VITABOX (e.g. an extra environmental sensor), as well as to define the VITABOX location used for geo-tracking in case of alarm events or to locate nearby pharmacies. The sponsor is responsible for registering patients associated with the VITABOX, define other users as caregivers, associate doctors to patients and define which devices (medical and environmental) will be used by each patient, allowing the system to scale not only in the home environment, but also in nursing homes or hospitals.

The provided web portal allows a user with a caregiver account to view historical data of his/her associated patients, which includes environmental and biometric information. Moreover, VITABOX and patients' data such as location and prescribed medication among others are also accessible, being helpful in settings where a caregiver follows/ assists multiple seniors in assisted homes or their homes.
The doctor may consult the biometric history of the patient and be automatically notified when the values fall out of the acceptable ranges. These notifications are triggered based on the clinical profile previously defined by the doctor (settings include minimum and maximum thresholds in different periods of the day). The doctor also has the possibility of scheduling the examinations to be performed periodically (including several schedules in the same day). A reminder is issued, appearing on the TV whenever the senior delays or fails to perform a given examination or measurement. The history of biometric data can be printed to pdf, producing a report that patients can take when attending a medical consultation with doctors who do not have access to the platform. Data can be as well exported to csv files for data analysis.

\section{Usability assessment methods}

The usability assessment of VITASENIOR-MT validates its relevance and potential use for the elderly, but also for the authorized formal and informal caregivers. For that purpose, two main components of the VITASENIOR-MT were considered:

- TV-based home-care system: usability assessment by direct end-users;

- Web portal: usability assessment by healthcare professionals.

This assessment was carried out at different times, first encompassing exploratory sessions to collect feedback from users in a UCD approach and then through systematic testing.

\subsection{Participants: sample characterization}

The assessment of the TV-based home-care system, in systematic tests, involved a senior group and a control group with 20 participants each (40 in total). Then, three participants from the senior group used the system for 2 months. The study and experiments were approved by the ethical committee of the Polytechnic Institute of Coimbra (ref. 28/2018). All participants from senior and control groups provided their informed consents.

The inclusion criteria of the senior group were: 1) age above 64 years; 2 ) no severe physical or mental disabilities limiting the use of the platform; and 3 ) reading skills. Participants were recruited from 2 sub-systems of the same institution, a Nursing home and an Assisted dwelling centre both from "Santa Casa da Misericórdia de Tomar", with the help of caregivers and nursing staff. It is noteworthy that most residents of the nursing home do not have minimum 
Table 1 Participants characteristics

\begin{tabular}{|c|c|c|}
\hline $\begin{array}{l}\text { Variable } \\
\text { (N=20 for each group) }\end{array}$ & $\begin{array}{l}\text { Senior group } \\
\text { (Number) }(\%)\end{array}$ & $\begin{array}{l}\text { Control group } \\
\text { (Number)(\%) }\end{array}$ \\
\hline Age & $80.0(\mathrm{SD} 8.63)$ & $48.3(\mathrm{SD} 10.64)$ \\
\hline \multicolumn{3}{|l|}{ Gender } \\
\hline Male & $10(50 \%)$ & $10(50 \%)$ \\
\hline Female & $10(50 \%)$ & $10(50 \%)$ \\
\hline \multicolumn{3}{|l|}{ Education } \\
\hline Primary school( $\leqslant 4$ years $)$ & $10(50 \%)$ & $0(0 \%)$ \\
\hline $\begin{array}{l}\text { Secondary school }(\geqslant 5 \text { and } \leqslant 12 \\
\text { years) }\end{array}$ & $8(40 \%)$ & $3(15 \%)$ \\
\hline University graduation & $2(10 \%)$ & $17(85 \%)$ \\
\hline \multicolumn{3}{|l|}{ Digital literacy } \\
\hline Smartphone, tablet or computer & $0(0 \%)$ & $20(100 \%)$ \\
\hline TV set and remote & $17(85 \%)$ & $20(100 \%)$ \\
\hline \multicolumn{3}{|l|}{ Physical impairments } \\
\hline Vision & $16(80 \%)$ & $11(55 \%)$ \\
\hline Hearing & $9(45 \%)$ & $0(0 \%)$ \\
\hline Motor & $9(45 \%)$ & $0(0 \%)$ \\
\hline
\end{tabular}

autonomy to live alone, having been excluded from the outset even before applying the exclusion criteria.

The first subgroup has 14 participants, aged between 65 and 89 years (average of 77.9 years), from the nursing home. Some of them are permanent residents, while others only stay in the nursing home during the day. The second subgroup, from the assisted dwelling centre, has 6 participants, aged between 79 and 92 years (average of 84.8 years), living autonomously in individual studios. Overall, the senior group is aged between 65 and 92 years (average $=80.0, \mathrm{SD}$ $=8.63$ ) and is equally divided by gender. Considering the education level, $50 \%$ of the participants have attended 4 or fewer years of school, $40 \%$ attended the secondary school (between 5 and 12 years), and 10\% have high-level graduation degrees. All participants reported that they do not use a smartphone, tablet or computer, but $85 \%$ watch TV regularly and know how to use the TV remote. Regarding physical impairments, $80 \%$ of the users have vision impairments not corrected by wearing glasses, and $45 \%$ of the users have hearing and some motor limitations affecting arm(s) or the hand(s) (e.g. arthrosis). The control group was composed of 20 participants, with ages between 25 and 67 years (average of 48.3 years, $\mathrm{SD}=10.64$ ), mostly recruited from the Polytechnic Institute of Tomar. The inclusion criteria were: 1) to be active professionally and 2) use regularly digital technologies such as computers or smartphones. This choice of participants aimed to allow us to compare the impact of digital literacy and age on the usability of the platform. Regarding their education levels, $15 \%$ have secondary education (12 years) and $85 \%$ have University graduation. They all regularly use their smartphone, tablet or computer, social networks, and also watch TV and use the remote. Regarding physical impairments, $55 \%$ of the participants of the control group reported vision limitations corrected to normal with glasses. Hearing and motor problems were not reported by any participant in the control group. Table 1 summarizes this information. Four health professionals (1 doctor and 3 nurses) assessed the web portal. They all reported that they use the computer several times a day and use professional healthcare software daily.

\subsection{TV-based Home-care system: usability tests}

Exploratory tests took place periodically during the development and optimization phases. These experiments were used to collect feedback from seniors, health professionals and invited experts. They took place in the research laboratory and in the facilities of the senior centres. Then, a set of systematic experiments was conducted with the senior group in the Nursing home and Assisted dwelling centre facilities, and with the control group in the research laboratory and in tech fairs. Each session began with a description of the study, followed by a demonstration of the operation of all parts of the system and finally an explanation of the test procedure. Before starting the test, each participant had the opportunity to try out the system and clarify any doubts with the researcher. Participants were then interviewed to be profiled regarding age, education, physical impairments (vision, hearing, etc.) and regarding their interaction with technologies, to infer, in particular, how often they watch TV, use mobile phones, computers, tablets, and Internet (including social networks). Participants not meeting the inclusion criteria were excluded. The experiments followed the same procedure for both groups, although the time to explain all the parts was much longer with the senior group. As soon as each participant felt ready, they were asked to perform $5 \mathrm{TV}$ interaction tests, called hereinafter as tasks, more specifically:

1. TASK 1: start the system by toggling from television broadcast mode to home-care system mode;

2. TASK 2: perform weight measurement;

3. TASK 3: check out the history of weight measurements;

4. TASK 4: check out the history of environmental sensor measurements;

5. TASK 5: perform blood pressure measurement assisted by audio-description.

The overall time for this experimental session ranged between 1.5 and 2 hours for the senior group (considering the initial explanation, application of questionnaires and interaction tests). The duration depended on the users' specificities, always respecting their own pace. 


\subsection{Web portal: usability tests}

Health professionals participated in the development loop, proposing new features and providing feedback regarding layouts, navigation options, type of and access to information of the web portal. They then monitored the 3 senior participants who used the platform regularly for 2 months. They started by defining clinical protocols/profiles (setting minimum and maximum thresholds) and scheduling examinations. During the 2-month evaluation period, they had to follow up patients, for example, visualize biometric history data, analyse notifications, and eventually adjust clinical profiles and send notification messages whenever necessary. Given the low number of health professionals participating in the experiments, nurses played the role of doctors.

\subsection{Evaluation methodology}

Two types of questionnaires were adopted to measure the subjective usability and user perceived satisfaction of the home-care system. First, a customized evaluation questionnaire was applied to evaluate the specificities of the homecare platform, regarding its different components (measurements with different medical devices, access to data history, etc). This questionnaire comprised 29 questions to evaluate the TV interaction quality, concerning ease of access, information understanding, provision and usefulness, rated from 1 to 5 (5-point Likert scale), where 1 is very bad and 5 is very good (this questionnaire is available in supplementary material). We also used a quantitative metric that consisted of measuring the time to perform the tasks. Additionally, the qualitative-validated questionnaire USE [34] was also adopted, generally assessing the subjective usability in 4 dimensions, namely Usefulness, Satisfaction, Ease of use and Ease of learning which comprises 30 items scored from 1 to 7 (1-strongly disagree and 7-strongly agree, all positive statements), though 3 statements were discarded as they did not apply. In comparison with the SUS (System Usability Scale) questionnaire, which is more commonly used, the USE questionnaire has the advantage of assessing the 4 dimensions separately, while SUS provides a single dimension score. Nevertheless, SUS and USE have shown to strongly correlate [35].

To assess the usability of the web portal, 4 health professionals ( 1 doctor and 3 nurses) answered a customized questionnaire specific to the web portal, which was inspired by Webqual 4.0 and USE, with 25 questions related to ease of use, interaction, information access, layout, functionality (scheduling, profiling, notifications and data history). This customized questionnaire is also available as supplementary material.

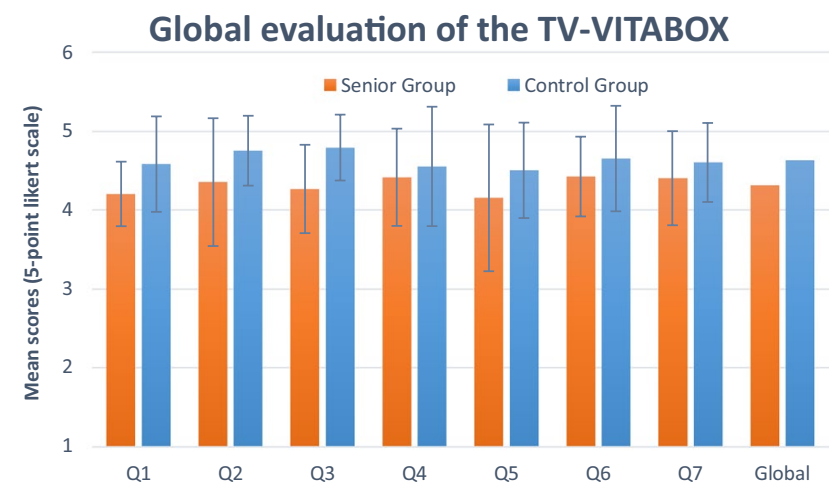

Fig. 3 Results of "TV-based home-care platform" customized questionnaire-global appreciation (5-point Likert scale). Average scores for questions $Q 1-Q 7$ (Q1 - Access to application, Q2-Usefulness, Q3-Font size, Q4- Information layout, Q5-Understanding of menus, Q6-Color, and Q7-Navigation on application). "Global" refers to the average of all questions scores

\section{Experimental results}

\subsection{Periodic tests during development}

Periodic exploratory tests occurring during the developing cycle were extremely useful. A significant amount of improvements has been achieved from perceived issues in user-system interaction, and from the suggestions provided by seniors, caregivers and health professionals, all contributing to a better overall solution. We mention just a few, for example, for the home-care platform: adjustment the reaction time of the TV remote control by software (seniors, in general, press the keys of the remote for a long time, leading to uncontrolled selections), highlight of the "help pictures and text" at the exact moment of audio description, adaptation of the help information content, change of items' colours and sizes, highlight of the 7 keys on the remote with a printed cover, and for the web portal, healthcare professionals proposed, for example: a redirect to a "notification page" as soon as they login (to get an immediate picture of abnormal situations), changes in layout and navigation between pages, definition of clinical profiles for different times of the day (morning and afternoon) to set different clinical thresholds, a more flexible way to schedule examinations and a direct communication channel with their patients.

\subsection{Home-care platform: customized questionnaires}

Figures 3 and 4 show a summary of the scores obtained from the customized questionnaire assessing the "TV-based home-care platform", by the senior centre group (SG) and the control group (CG). Figure 3 reflects the global assessment regarding the following questions: $Q 1-$ Access to 
Fig. 4 Results of "TV-based home-care platform" customized questionnaire-specific appreciation (5-point Likert scale). Average scores for questions W1-W3 (W1-Usefulness, W2-Easiness, W3-Duration) and $B P 1-B P 3(B P 1-U s e f u l-$ ness, BP2-Easiness, BP3Duration; $W$ and $B P$ stand, respectively, for "weight" and "blood pressure" physical examinations, and G1-G3 (G1-User selection, G2-Exam and history access tabs, and G3Audio-description)

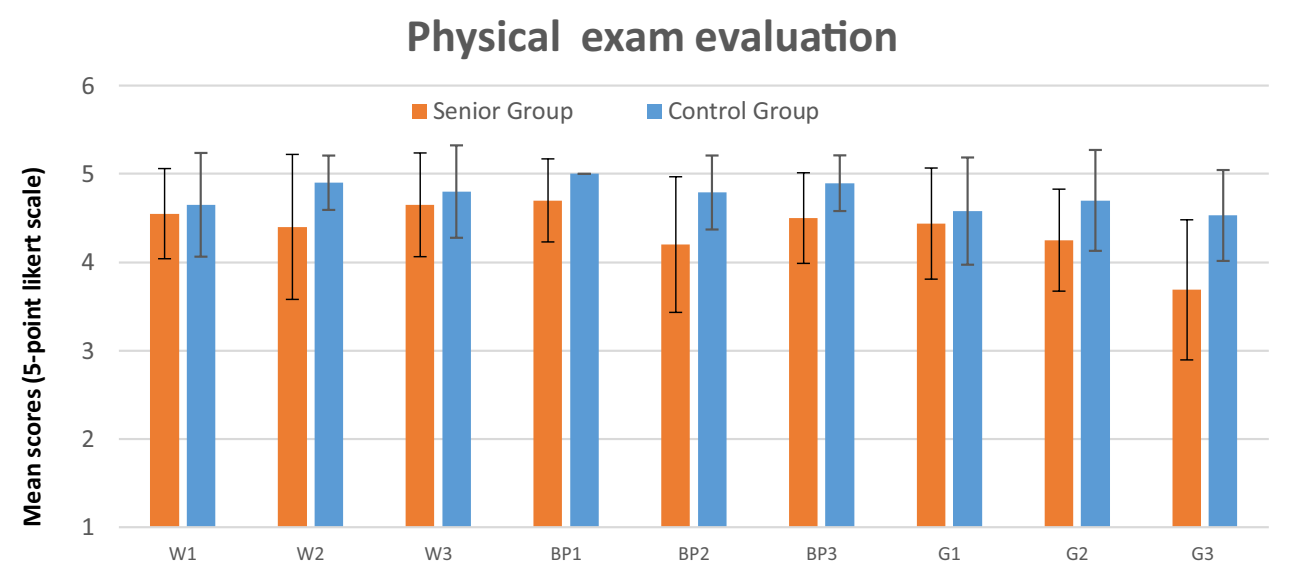

application (specific to TASK 1), Q2-Usefulness, Q3Font size, Q4-Information layout, Q5-Understanding of menus, Q6-Color, and Q7-Navigation on application $(Q 2$ to $Q 7$ globally evaluating the four remaining tasks). Results show a mean Global score of 4.3 and 4.6, respectively, for SG and CG. Both scores are high, although there is a slight difference between groups (statistically significant, t-test two-tail, $p=0.019$ ), which attest the overall good impression about the system. The lowest scores were obtained for Q5-Understanding of menus, in both groups, respectively, 4.2 and 4.5 for SG and CG. On the other hand, the highest scores were obtained in Q6-Color and Q3-Font size, respectively, for SG and CG. The CG rated $Q 1, Q 4$, $Q 5$ below the global score, while the $\mathrm{SG}$ rated $Q 1, Q 3, Q 5$ below the global score.

Figure 4 shows the evaluation results for TASK 2 (questions W1-Usefulness, W2-Easiness, W3-Duration) and TASK 5 (questions BP1-Usefulness, BP2-Easiness, BP3Duration), specifically assessing the execution of physical examinations (W-weight and $\mathrm{BP}$ - blood pressure). Questions G1-User selection, G2-Exam and history access tabs and G3-Audio-description assess users' satisfaction while performing TASKS 2,3,4,5. Both groups have considered that user selection is easy, presenting a very small score difference between groups, 4.4 and 4.6, respectively, for SG and CG. The CG scored higher the access to Exam and history tabs, and audio-description than the SG. Special emphasis is given to audio-description, with a difference of 0.84 between the 2 groups. Both groups expressed blood pressure measurement as more useful than weight measurement. On the other hand, both groups scored the weight examination as being easier than the blood pressure examination, which is understandable as the use of the weight scale is very simple. The results show the largest assessment difference between the 2 groups in the ease of use, with the CG performing better than the SG. This result is supported by Fig. 5, which shows the average time required to perform the 5 tasks. There is a large difference in execution time between the 2 groups for all tasks, in particular, for the weight measurement. Despite these results, the SG assessed the perceived time to perform the examination not very differently of the CG (questions $W 3$ and BP3 in Fig. 4), which means that they considered the time as normal (i.e. the time they usually take with their own devices).

To find out whether there was a correlation between the execution time and age and education, a Pearson correlation was applied between these variables (see Table 2). Results show that there is no statistically relevant correlation for execution time vs education for both groups, but there is a correlation between execution time vs. age for the CG. These results show that both age and education of the SG did not influence participants' performance.

\subsection{Home-care platform: USE evaluation}

The responses to the USE questionnaire evaluated four metrics: ease of use, ease of learning, overall satisfaction and usefulness (see Fig. 6), in a 7-point Likert scale. Results show that there are no significant differences in the evaluation of satisfaction and usefulness by both groups. On average, the SG senior scored satisfaction with 5.7 and usefulness with 5.8, while the CG evaluated these items with 5.8 and 6.0, respectively. The greatest differences among the

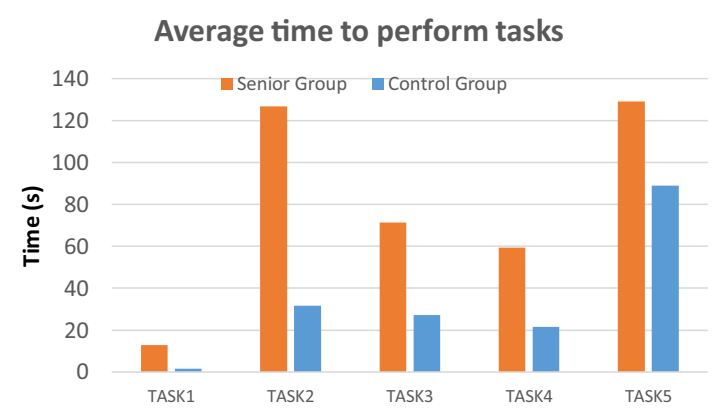

Fig. 5 Average time (in seconds) required to accomplish each task 


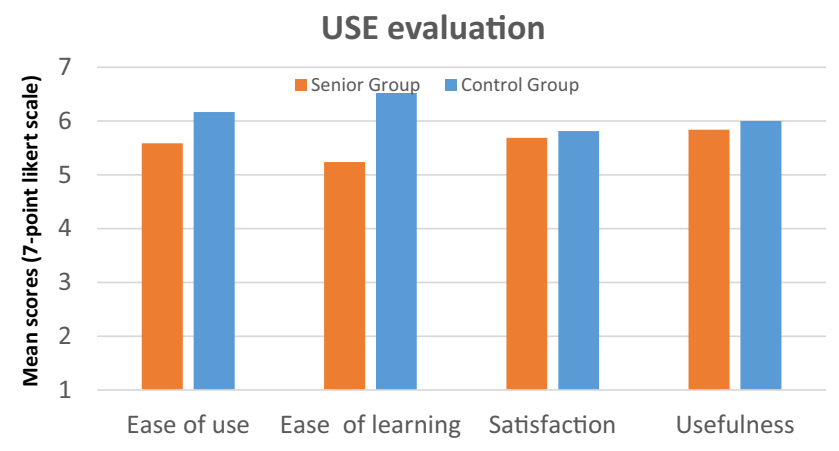

Fig. 6 Results of USE qualitative-validated questionnaire (7-point Likert scale)

Table 2 Correlation (Pearson) to assess correlations between age and execution time, age and satisfaction and education and execution time

\begin{tabular}{llll}
\hline & Time/age & Satisfaction/age & Time/education \\
\hline Senior & 0.15 & -0.12 & -0.30 \\
Control & $0.57 *$ & 0.11 & 0.15 \\
\hline
\end{tabular}

*statistically relevant (Pearson Correlation coefficient $r>0.5$ )

mean scores of the two groups occur for the ease of use and ease of learning items, respectively, 5.6 and 5.2, and 6.2 and 6.5, for SG and CG. The difference in results is not surprising, as learning skills decline with age and are closely related to whether or not a person is professionally active.

Table 2 shows that there is no correlation between satisfaction and age for both groups, which emphasizes the good level of satisfaction with the platform regardless of age.

\subsection{Long-term evaluation: Web portal and home-care platform}

Three senior participants selected from the senior group used the home-care platform for a period of 2 months. Evaluation of the system was carried out just after this period. Patient 1 (P1) was a hypertensive patient, under anti-hypertensive treatment (comorbidities: epilepsy, arthritis and paroxistic atrial fibrillation), Patient 2 (P2) was an hypertensive patient and stroke survivor, under anti-hypertensive treatment, and Patient 3 (P3) was an hypertensive diabetic patient, under anti-hypertensive and diabetes treatment. Patients P1, P2 and P3 measured regularly blood pressure, heart rate and weight, and patient $\mathrm{P} 3$ measured additionally glucose levels. The frequency of measurements was set by the doctor and nurses (most measurements were made on a daily basis). Patients used the home-care platform to perform the examinations, according to scheduling. During this period, the research team provided help in clarifying procedures whenever requested, and had regular contact with health professionals. At the end of the 2-month period, health professionals answered the web portal assessment questionnaire regarding their interaction with the web portal. Given the small sample, we did not perform a statistical analysis, however it is worth noting that almost all usability items were scored at maximum. Biometric collected data were analysed by the health professionals, who calculated the mean SBP (systolic blood pressure) and DBP (dystolic blood pressure) and visit-to-visit BP to infer abnormalities, and analysed weight trends. A case study reporting the added-value of the system is presented in [36]. Senior participants reported that they were satisfied with the usefulness of the system and that they used it without difficulty, showing interest in continuing to use it.

\section{Discussion}

This study aimed to evaluate the usability of the interaction with TV on the proposed healthcare platform VITASENIOR-MT. The usability scores obtained from subjective questionnaires were high (global appreciation equal to 4.3 (SG) and 4.6 (CG) and specific appreciation of the execution of physical examinations all above 4 for both groups except the audio-description in SG, in a 5-point scale customized questionnaire; all scores were above 5 for both groups in the 4 dimensions of the USE questionnaire in a 7-point scale), which confirms that older adults were satisfied and able to use the TV remote efficiently to perform physical examinations and access data. Although the CG generally scored better for the platform, the differences in scores between the two groups were small for most items, which emphasizes the usability of the platform independently of users' profile.

Looking at the items that had a larger difference between the two groups, we observe that they are all related to motor, sensory and cognitive issues, as described next. In the global score (Fig. 3), the "font size" evaluation showed the greatest difference between the 2 groups, which may be explained by the lower visual acuity affecting the SG. The audio-description also received the largest score difference (Fig. 4), which might be related to hearing loss as reported by several SG participants, or in more difficulty in dealing simultaneously with multisensory cues (visual and auditory). However, both the font size and the audio level can easily be improved thanks to the TV's large screen size and audio amplifier. The execution time to perform the tasks was much longer in the SG as observed in Fig. 5, which results from the lowest motor weaknesses and coordination (e.g. mobility and dexterity) of the SG, but also the longer time to "think about the action to take" due to decline on executive functions [37]. The largest difference was observed for the weight measurement, as many of the senior participants took a significant amount of time to stand up and to step on 
the scale. Correlation analysis between age and execution time showed to be statistically significant for the CG but not for the SG. This corroborates the idea of a decline in motor and cognitive skills that affects the elderly above a certain age, which is more visible here because the average age of the SG participants is quite high (80 years). These results provide quantitative data on how the use of TV interaction may represent an important asset in comparison to tablets and smartphones, for which the negative effect of sensory and motor limitations would be much steeper. The ease of learning in the USE questionnaire (Fig. 6) was the worst dimension scored by the SG and with the largest difference between the 2 groups. This is explained by the decline in cognitive performance due to age [37], for example, working memory, and is well evidenced by the item that obtained the lowest score (4.7 in 1-7 scale) in this dimension: "I easily remember how to use it". This very limiting issue for the elderly highlights the need to design the interaction procedures as simple as possible to be easily remembered.

The non-correlation between "execution time" and "education" and between "satisfaction" and "age" shows that the interaction with the system was affected mainly by users' physical impairments and not by their literacy and that users' age did not influence their level of satisfaction, which supports the acceptance of the platform.

Although it is a very familiar and easy-to-use device for the elderly, the research of TV interaction as a means of providing healthcare services is still scarce. The achieved results show that this is a viable solution, even though the sensory and cognitive deficits of the elderly have been shown to affect the interaction. It is worth remembering that in the interaction process of the proposed solution, there were basically 7 keys of the remote and 3 steps to memorize, namely selecting the type of task (e.g. perform examination or visualize data), selecting the participant and selecting the examination. Thus, the platform has been designed to minimize seniors' effort. Although the visual layout and widget placement of the user interface followed a standard approach, it was adjusted according to user feedback. Nevertheless, other interaction approaches can be researched following the "one key - one function" and "one page - one task" paradigms [38], for example, to further minimize the number of items to memorize, the steps to be performed or the number of remote's keys to use. The level of digital literacy is of course an additional factor that affects the learnability of the interaction, as people who have never used a computer or a smartphone in their lives have no automated mental processes to navigate in the menus. In the current study, none of the SG participants have reported ever using a smartphone or tablet which enforces the idea that these devices might not be a solution for most older adults, although most proposed approaches are based on mobile applications. Another important observation that came up from the informal conversations with the elderly, related to socio-cultural issues, is the rejection of the technology in anticipation. Although using it efficiently and stating that it would be a useful platform, some seniors mentioned "This is not for me", which expresses the idea that technology is not for them, since they never used technology in their lives. Here, the role of health professionals, particularly doctors, can be important to change that mindset by "prescribing"/ recommending the platform as a medication, as elderly patients trust heavily on doctors [6]. Actually, remote patient monitoring is a promising contributor to warrant universal health coverage, acknowledged as a major goal by all the Member States of the World Health Organization.

Finally, the good evaluation results of the platform resulted in part from the user-in-the-loop design that we followed, where we received feedback from direct end-users and health professionals, which allowed us to change or improve many issues before proceeding to the systematic usability tests and the longitudinal tests. The stable hardware and software free of bugs, and the seemingly way that end-users operated different medical devices using the same interaction process, all contributed to the level of acceptance of the proposed solution. The usability evaluation results provide quantitative data that supports the viability of the VITASENIOR-MT solution based on TV interaction, in a population of senior participants with low digital literacy and education, which shows its potential adoption by the elderly as a consumer product for health. This stresses out that technology can be adopted by potential users as long as it offers characteristics of customization and universality. Therefore, human-computer interaction processes should be, sometimes, simplified to ensure universal access. From a hardware and data connectivity perspective, VITASENIOR-MT also presents some interesting features compared to other similar platforms, which are important for its adoption. Namely, 1) the solution is independent of the digital television network and operator and 2) the telehealth service can work without a continuous Internet link, since VITABOX is independent of television services. This is quite important, as user experience is not affected by network conditions and bandwidth, which may be particularly relevant in remote rural areas.

\subsection{Limitations of the study and future work}

Despite the good evaluation results obtained in systematic testing, the group of 20 participants recruited from a Nursing home and an Assisted dwelling centre, both from "Santa Casa da Misericórdia de Tomar" (a national Portuguese Private Institution of Social Solidarity) may not be a representative sample of the target senior population. The technology acceptance has been shown to be very dependent of users' profile [6]. Therefore, the experimental tests 
should go beyond nursing homes to broaden the spectrum of participants. The number of participants in the longitudinal tests was also very small, not allowing a statistical analysis, and they were carried out in a controlled environment (assisted dwelling centre). For example, it was not possible to evaluate how the use of the health platform may have disrupted their regular way of watching TV, as seniors used the community TV to watch TV and the TV in their rooms only for use as telehealth platform. Testing the solution in home-settings and involving the follow-up of family doctors is required to assess the effective acceptance and utility of the solution. Nevertheless, the highly positive appreciation of the VITASENIOR-MT platform by both senior end-users and health professionals is undoubtedly a good indicator of the telehealth platform's potential. This is also recognized by a Technology transfer agreement that was achieved with a telehealth company that is soon starting the commercialization of a solution based on VITASENIOR-MT, thus complementing a wearable monitoring device that the company currently commercializes.

\section{Conclusion}

VITASENIOR-MT is a telehealth solution that has been designed specifically to address usability issues fitting the needs of all actors in the system, and specially older adults. Overall results show that the levels of Ease of use, Ease of learning, Satisfaction and Usefulness of the home-care platform, validated by a senior group and a control group, were high, demonstrating the viability of TV interaction for the target population, with task performance and overall scores of the senior group approaching those of the control group. Additionally, it has been shown that, for the senior group, old age and low digital literacy and education are not statistically relevant in terms of the system usability and that the sensory deficits are the most limitative issues on the interaction. Most telehealth studies and implementations often disregard usability issues, or do not include them as part of the development phase, leading to systems that end up being a barrier to their adoption. Thus, the proposed solution is an important contribution and asset for the telehealth systems universe. Long-term testing has been conducted with a small number of participants and in a semi-controlled environment and need to be extended, but showed the potential benefit of the solution for healthcare follow-up.

\section{Supplementary Material}

In the link http://orion.ipt.pt/ gpires/VITASENIOR-MT/ videos/videos.htm, the following supplementary material is provided: home-care tasks, home-care customized questionnaire, home-care USE questionnaire, customized web portal questionnaire, demonstration videos of homecare interaction and video of web portal interaction.

Acknowledgements The authors would like to thank all seniors and staff (board, caregivers, health professionals) of the Nursing home and Assisted Homes of "Santa Casa da Misericordia de Tomar" (SCMT), all participants of the control group, and all invited experts, for their time, suggestions and contributions.

\section{Declarations}

Conflict of interest The authors report no conflicts of interest.

\section{References}

1. Commission, E.: Reflection paper: towards a sustainable europe by 2030 (2019)

2. OECD/EU: Health at a glance: Europe 2018 state of health in the eu cycle. OECD Publishing (2018)

3. MJ, A., IM, E.E., DD, K.: A review on the technologies and services used in the self-management of health and independent living of elderly. Technol. Health Care 22(5), 677:87 (2014)

4. Karlsen, C., Ludvigsen, M., Moe, C., K, H., Thygesen, E.: Experiences of community-dwelling older adults with the use of telecare in home care services: a qualitative systematic review. JBI Database Syst. Rev. Implement. Rep. 15(12), 2913-2980 (2017)

5. Guner, H., Acarturk, C.: The use and acceptance of ict by senior citizens: a comparison of technology acceptance model (TAM) for elderly and young adults. Univ. Access Inf. Soc. 19, 311-330 (2020)

6. LeRouge, C., Ma, J., Sneha, S., Tolle, K.: User profiles and personas in the design and development of consumer health technologies. Int. J. Med. Inf. 82(11), e251-e268 (2013)

7. Blandford, A.: Hci for health and wellbeing: Challenges and opportunities. Int. J. Human Comput. Stud. 50 years of the International Journal of Human-Computer Studies. Reflections on the past, present and future of human-centred technologies 131, 41 - 51 (2019)

8. Deldar, K., Bahaadinbeigy, K., Tara, S.M.: Teleconsultation and clinical decision making: a systematic review. Acta Informatica Medica AIM J. Soc. Med. Inf. Bosnia Herzegovina 24(4), 286292 (2016)

9. Otto, C., Shemenski, R., Drudi, L.: Real-time tele-echocardiography: diagnosis and management of a pericardial effusion secondary to pericarditis at an antarctic research station. Telemed. e-Health 18(7), 521-524 (2012)

10. Klemets, J., Maattala, J., Hakala, I.: Integration of an in-home monitoring system into home care nurses' workflow: a case study. Int. J. of Medical Inf. 123, 29-36 (2019)

11. Hung, L.P., Lin, C.C.: A multiple warning and smart monitoring system using wearable devices for home care. Int. J. Human Comput. Stud. 136, 102381 (2020)

12. Majumder, S., Aghayi, E., Noferesti, M., Memarzadeh-Tehran, H., Mondal, H., Pang, Z., Deen, M.: Smart homes for elderly healthcare-recent advances and research challenges. Sensors 17(11), 2426 (2017)

13. Islam, S.M.R., Kwak, D., Kabir, M.H., Hossain, M., Kwak, K.: The internet of things for health care: a comprehensive survey. IEEE Access 3, 678-708 (2015) 
14. Ali, E.E., Chew, L., Yap, K.Y.L.: Evolution and current status of mhealth research: a systematic review. BMJ Innov. 2(1), 33-40 (2016)

15. Foster, M., Sethares, K.: Facilitators and barriers to the adoption of telehealth in older adults: an integrative review. CIN Comput. Inf. Nurs. 32(11), 523-533 (2014)

16. Padilla-Gongora, D., Lopez-Liria, R., del Pilar Daz-Lopez, M., Aguilar-Parra, J.M., Vargas-Munoz, M.E., Rocamora-Perez, P.: Habits of the elderly regarding access to the new information and communication technologies. Proc. Soc. Behav. Sci. Education, Health and ICT for a Transcultural World 237, 1412-1417 (2017)

17. Cid, A., Sotelo, R., Leguisamo, M., Ramirez-Michelena, M.: Tablets for deeply disadvantaged older adults: Challenges in longterm care facilities. Int. J. Human Comput. Stud. 144, 102504 (2020)

18. Bujnowska-Fedak, M.M., Grata-Borkowska, U.: Use of telemedicine-based care for the aging and elderly: promises and pitfalls. Smart Homecare Tech. TeleHealth 2015(3), 91-105 (2015)

19. Seto, E., Leonard, K.J., Masino, C., Cafazzo, J.A., Barnsley, J., Ross, H.J.: Attitudes of heart failure patients and health care providers towards mobile phone-based remote monitoring. J. Med. Internet. Res. 12(4), e55 (2010)

20. Silva, T., Caravau, H., Campelo, D.: Information needs about public and social services of portuguese elderly. In: International Conference on Information and Communication Technologies for Ageing Well and e-Health, pp. 46-57. Porto (2017)

21. Andreadis, A., Zambon, R., Parlangeli, O.: Tv as an experience conveyer for better acceptance of ict services by older adults. Univ. Access Inf. Soc. pp. 1615-5297 (2020)

22. Normie, L.: Technology for ageing in place. IFA Global Age. 7, 45-53 (2011)

23. Ribeiro, V.S., Martins, A.I., Queiros, A., Silva, A.G., Rocha, N.P.: Usability evaluation of a health care application based on iptv. Proc. Comput. Sci. 64, 635-642 (2015)

24. Macis, S., Loi, D., Ulgheri, A., Pani, D., Solinas, G., La Manna, S., Cestone, V., Guerri, D., Raffo, L.: Design and usability assessment of a multi-device soa-based telecare framework for the elderly. IEEE J. Biomed. Health Informat. pp. 1-1 (2019)

25. Costa, C.R., Anido-Rifon, L.E., Fernandez-Iglesias, M.J.: An open architecture to support social and health services in a smart tv environment. IEEE J. of Biom. Health Info. 21(2), 549-560 (2017)

26. Silva, T., Caravau, H., de Abreu, J.F., Reis, L.: Seniors infoinclusion through interactive television: results of a field trial. In: 4th International Conference on Information and Communication Technologies for Ageing Well and e-Health, pp. 134-141 (2018)
27. Epelde, G., Valencia, X., Abascal, J., Daz, U., Zinnikus, I., Husodo-Schulz, C.: Tv as a human interface for ambient intelligence environments. In: 2011 IEEE International Conference on Multimedia and Expo, pp. 1-6 (2011). https://doi.org/10.1109/ ICME.2011.6012186

28. Spinsante, S., Gambi, E.: Remote health monitoring by osgi technology and digital tv integration. IEEE Trans. Consumer Electron. 58(4), 1434-1441 (2012)

29. Santana-Mancilla, P.C., Anido-Rifon, L.E.: The technology acceptance of a tv platform for the elderly living alone or in public nursing homes. Int. J. Environ. Res. Public Health 14(6:617) (2017)

30. Independa: https://independa.com/ [Accessed March 2021] (2020)

31. Elio: https://e-lio.fr/e-liobox [Accessed March 2021] (2020)

32. Pires, G., Correia, P., Jorge, D., Mendes, D., Gomes, N., Dias, P., Ferreira, P., Lopes, A., Manso, A., Almeida, L., Oliveira, L., Panda, R., Monteiro, P., Gracio, C., Pereira, T.: VITASENIORMT: A telehealth solution for the elderly focused on the interaction with TV. In: IEEE 20th International Conference on e-Health Networking, Applications and Services (Healthcom), pp. 1-6 (2018)

33. Mendes, D., Panda, R., Dias, P., Jorge, D., Antonio, R., Oliveira, L., Pires, G.: VITASENIOR-MT: A distributed and scalable cloud-based telehealth solution. In: 2019 IEEE 5th World Forum on Internet of Things, Limerick, Ireland, pp. 1-6 (2019)

34. Lund, A.M.: Measuring usability with the use questionnaire. Usability Interface 8(2), 3-6 (2001)

35. Gao, M., Kortum, P., Oswald, F.: Psychometric evaluation of the use (usefulness, satisfaction, and ease of use) questionnaire for reliability and validity. Proc. Human Factors Ergonom. Soc. Annu. Meeting 62(1), 1414-1418 (2018)

36. Pereira, T., Pires, G., Jorge, D., Santos, D.: Telehealth monitoring of a hypertensive elderly patient with the new vitasenior-mt system: a case study. Blood Pressure Monit. 25(4), 227-230 (2020)

37. Murman, D.L.: The impact of age on cognitions. Semin Hear. 36(3), 111-121 (2015)

38. Phiriyapokanon, T.: Is a big button interface enough for elderly users? In: Master Thesis in Computer Engineering, Malardalen University (2011)

Publisher's Note Springer Nature remains neutral with regard to jurisdictional claims in published maps and institutional affiliations. 